\title{
PA-073 BASELINE BACTERIAL LOAD AND RIFAMPICIN EXPOSURE ARE ASSOCIATED TO CULTURE CONVERSION IN A TWO-MONTH STUDY OF TUBERCULOSIS
}

Nyanda Ntinginya, ${ }^{1}$ Anna Framhein, ${ }^{2}$ Stella Mpagama, ${ }^{3}$ Hadija Semvua, ${ }^{4}$ Gibson Kibiki, ${ }^{5}$ Leonard Maboko, ${ }^{1}$ Michael Hoelscher, ${ }^{2}$

Norbert Heinrich ${ }^{2}$. NIMR-MMRC, Tanzania; ${ }^{2}$ DITM, Medical Center, LMU, Germany; ${ }^{3} \mathrm{KIDH}$, Tanzania; ${ }^{4} \mathrm{KCMC}$, Tanzania; ${ }^{5}$ East African Health and Research Commission, Burundi

\subsection{6/bmjgh-2016-000260.105}

Background Early bactericidal activity (EBA) during the first two weeks of TB treatment is an important method for early efficacy evaluation of new anti-tuberculosis agents.

Methods We performed an observational; two-site clinical study in Tanzania in patients with newly diagnosed pulmonary TB during the first eight weeks of standard HRZE treatment. Baseline and treatment-related covariates including X-ray, baseline bacterial load and rifampicin pharmacokinetics were analysed for their correlation to treatment success.

Results From Nov 2011 to July 2013 we enrolled 74 pulmonary TB patients from Moshi (41) and Mbeya (33). Mbeya participants had a higher baseline bacterial load measured by $\log$ time to positivity (TTP) in the MGIT culture system (median 1.29; IQR 1.09-1.46 vs 1.58; IQR 1.44-1.87; p< 0.001) Overall, 56/68 (80\%) of patients achieved a negative solid media culture, and 28/59 (47\%) achieved a negative liquid culture at 8 weeks. Median time to negative on LJ culture was 45.5 days (IQR 21-56), in liquid culture 56 days. The strongest association with outcome for any covariate was found for baseline bacterial load: patients with a positive week 8 LJ culture had a median $\log$ TTP of 1.20 (IQR 0.94-1.35); patients with a negative week 8 culture had $1.48(1.29-1.73 ; \mathrm{p}=0.006)$. In exploratory analysis, rifampicin area under the concentration curve (AUC) was associated with shorter time to LJ culture conversion in patients who achieved negative culture, (hazard ratio $1.05, \mathrm{p}=0.038$ ), but not in the total population.

Conclusions This observation EBA study using standard HZRE was successfully implemented with methodologies thus far established for the first time at the two Tanzanian sites. Baseline bacterial load was confirmed as an important predictive parameter. 